\title{
Nitrogen-Linked Diphosphine Ligands with Ethers Attached to Nitrogen for Chromium Catalyzed Ethylene Tri- and Tetramerizations
}

Paul R. Elowe, ${ }^{\dagger}$ Cassandra McCann ${ }^{\neq}$Paul G. Pringle, ${ }^{\neq}$Stefan K. Spitzmesser, $" \quad$ John E. $\operatorname{Bercaw}^{*}+$

$\dagger$ Arnold and Mabel Beckman Laboratories of Chemical Synthesis, California Institute of Technology, Pasadena, California 91125, ${ }^{\ddagger}$ School of Chemistry, University of Bristol, Bristol BS8 ITS, UK, and "Ineos, Rue de Ransbeek 310, 1120 Brussels, Belgium.

To whom correspondence should be addressed. E-mail: bercaw@ caltech.edu.

S1. Structural drawings, tables with structural parameters, and crystallographic information for $\mathbf{5}$. 


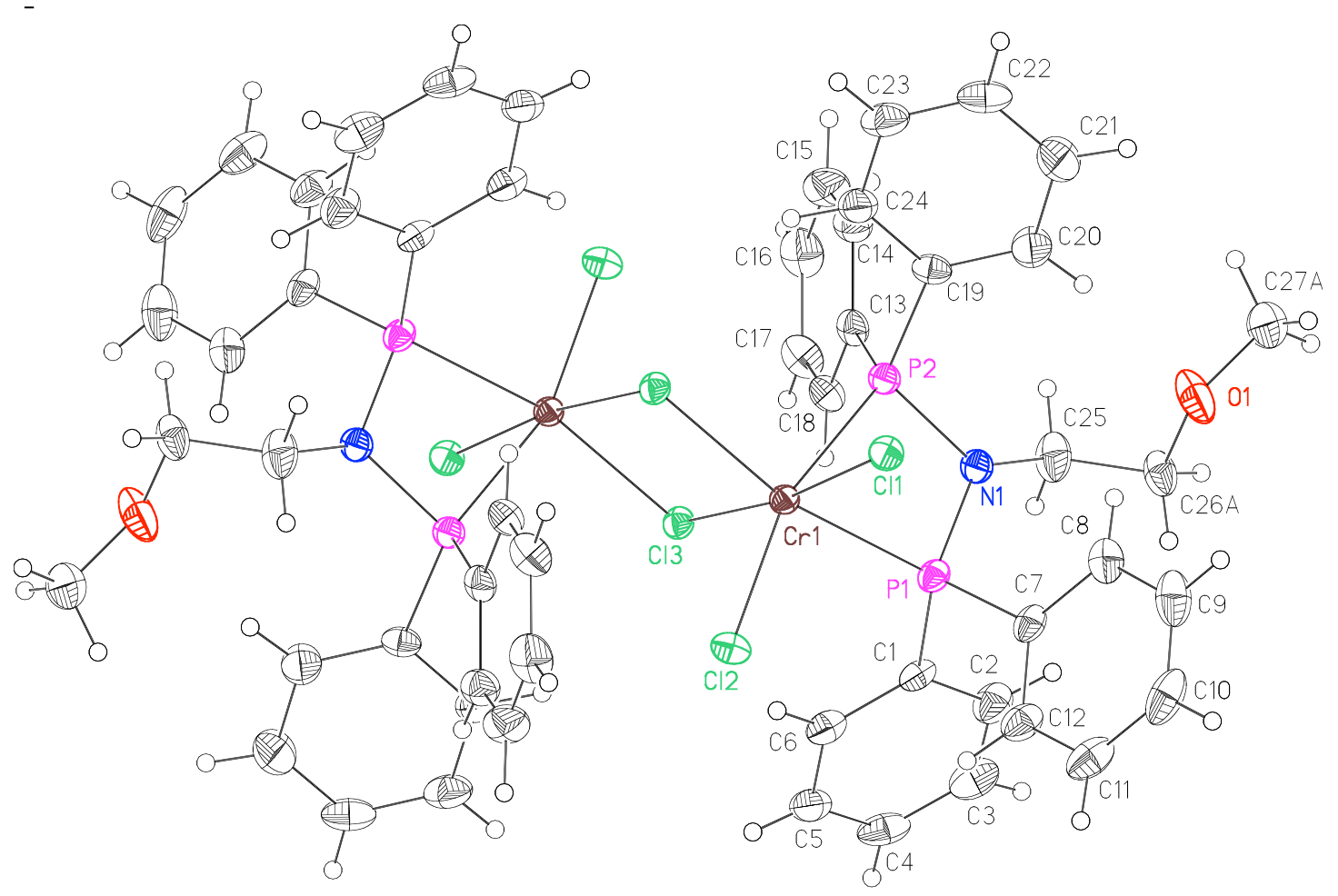

Figure 1. Structural drawing of $\mathbf{5}$ with thermal ellipsoids at the $50 \%$ probability level.

Table 1. Crystal data and structure refinement for 5 (CCDC 258068).

Empirical formula

Formula weight

Crystallization Solvent

Crystal Habit

Crystal size

Crystal color

Type of diffractometer

Wavelength

Data Collection Temperature

$\theta$ range for 19148 reflections used

in lattice determination

Unit cell dimensions

Volume

Z

Crystal system

Space group

Density (calculated)

$\mathrm{F}(000)$

$\theta$ range for data collection

Completeness to $\theta=33.74^{\circ}$

Index ranges

Data collection scan type
$\mathrm{C}_{54} \mathrm{H}_{54} \mathrm{Cl}_{6} \mathrm{~N}_{2} \mathrm{O}_{2} \mathrm{P}_{4} \mathrm{Cr}_{2} \bullet 4\left(\mathrm{CH}_{2} \mathrm{Cl}_{2}\right)$

1543.28

Dichloromethane/petroleum ether

Block

$0.41 \times 0.16 \times 0.15 \mathrm{~mm}^{3}$

Sapphire blue

\section{Data Collection}

\section{Bruker SMART 1000}

$0.71073 \AA \mathrm{MoK} \alpha$

100(2) K

\subsection{9 to $33.48^{\circ}$}

$\mathrm{a}=11.3376(5) \AA$

$\mathrm{b}=18.5701(7) \AA$

$\mathrm{c}=16.7264(7) \AA$

$\beta=108.7220(10)^{\circ}$

$3335.2(2) \AA^{3}$

2

Monoclinic

$\mathrm{P} 2{ }_{1} / \mathrm{n}$

$1.537 \mathrm{Mg} / \mathrm{m}^{3}$

1572

1.69 to $33.74^{\circ}$

$90.1 \%$

$-16 \leq \mathrm{h} \leq 16,-28<\mathrm{k}<28,-25<1<25$

$\omega$ scans at $3 \phi$ settings of $2 \theta=-28^{\circ}$ and 2 at $2 \theta=-40^{\circ}$ 
Reflections collected Independent reflections

Absorption coefficient Absorption correction Max. and min. transmission Structure solution program Primary solution method Secondary solution method Hydrogen placement

Structure refinement program

Refinement method

Data / restraints / parameters

Treatment of hydrogen atoms

Goodness-of-fit on $\mathrm{F}^{2}$

Final R indices [I $>2 \sigma(\mathrm{I}), 7974$ reflections]

$\mathrm{R}$ indices (all data)

Type of weighting scheme used

Weighting scheme used

Max shift/error

Average shift/error

Largest diff. peak and hole
54219

$12015\left[\mathrm{R}_{\mathrm{int}}=0.0669\right]$

$1.026 \mathrm{~mm}^{-1}$

None

0.8614 and 0.6785

SHELXS-97 (Sheldrick, 1990)

Direct methods

Difference Fourier map

Difference Fourier map

SHELXL-97 (Sheldrick, 1997)

Full matrix least-squares on $\mathrm{F}^{2}$

12015 / 0 / 491

Unrestrained, disordered riding

1.596

$\mathrm{R} 1=0.0458, w \mathrm{R} 2=0.0749$

$\mathrm{R} 1=0.0776, w \mathrm{R} 2=0.0783$

Sigma

$w=1 / \sigma^{2}\left(\mathrm{Fo}^{2}\right)$

0.002

0.000

1.183 and -0.759 e. $\AA^{-3}$

\section{Special Refinement Details}

There is disorder in the methyl-ethyl keytone ligand attached to nitrogen. The disorder was modeled with alternate positions for the ethyl carbon atom alpha to oxygen $(\mathrm{C} 26 \mathrm{~A} \& \mathrm{~B})$ with the corresponding changes in the methyl carbon (C27A \& B). The oxygen atom (O1) was refined at a single position. The molecule sits on a center of symmetry, therefore, only the unique atoms are labeled in the figures. Disorder is also observed in the dichloromethane solvents (not shown in the figures).

Refinement of $\mathrm{F}^{2}$ against ALL reflections. The weighted $\mathrm{R}$-factor $(w \mathrm{R})$ and goodness of fit $(\mathrm{S})$ are based on $\mathrm{F}^{2}$, conventional R-factors $(R)$ are based on $F$, with $F$ set to zero for negative $F^{2}$. The threshold expression of $F^{2}>2 \sigma\left(F^{2}\right)$ is used only for calculating R-factors(gt) etc. and is not relevant to the choice of reflections for refinement. R-factors based on $\mathrm{F}^{2}$ are statistically about twice as large as those based on F, and R-factors based on ALL data will be even larger.

All esds (except the esd in the dihedral angle between two l.s. planes) are estimated using the full covariance matrix. The cell esds are taken into account individually in the estimation of esds in distances, angles and torsion angles; correlations between esds in cell parameters are only used when they are defined by crystal symmetry. An approximate (isotropic) treatment of cell esds is used for estimating esds involving 1.s. planes. 
Table 2. Atomic coordinates $\left(x \mathbf{1 0}^{4}\right)$ and equivalent isotropic displacement parameters $\left(\AA^{2} \mathbf{x} \mathbf{1 0}^{3}\right)$ for 5 (CCDC 258068). U(eq) is defined as the trace of the orthogonalized $U^{i j}$ tensor.

\begin{tabular}{|c|c|c|c|c|c|}
\hline & $\mathrm{x}$ & $\mathrm{y}$ & $\mathrm{z}$ & $\mathrm{U}_{\mathrm{eq}}$ & Occ \\
\hline $\operatorname{Cr}(1)$ & $10258(1)$ & $5437(1)$ & $9145(1)$ & $16(1)$ & 1 \\
\hline $\mathrm{Cl}(1)$ & 9270(1) & 6364(1) & 8313(1) & 21(1) & 1 \\
\hline $\mathrm{Cl}(2)$ & 11971(1) & 6091(1) & 9890(1) & $23(1)$ & 1 \\
\hline $\mathrm{Cl}(3)$ & $11000(1)$ & 4392(1) & 9962(1) & $18(1)$ & 1 \\
\hline $\mathrm{P}(1)$ & $11095(1)$ & $5139(1)$ & $8022(1)$ & 19(1) & 1 \\
\hline $\mathrm{P}(2)$ & 8871(1) & $4635(1)$ & $8053(1)$ & $18(1)$ & 1 \\
\hline $\mathrm{O}(1)$ & 8632(1) & $4810(1)$ & $5551(1)$ & $42(1)$ & 1 \\
\hline $\mathrm{N}(1)$ & $9828(1)$ & $4661(1)$ & $7445(1)$ & $20(1)$ & 1 \\
\hline $\mathrm{C}(1)$ & $12508(2)$ & 4597(1) & $8274(1)$ & 20(1) & 1 \\
\hline$C(2)$ & $12868(2)$ & $4222(1)$ & 7671(2) & $30(1)$ & 1 \\
\hline$C(3)$ & 13952(2) & 3818(1) & 7916(2) & $34(1)$ & 1 \\
\hline$C(4)$ & $14698(2)$ & $3807(1)$ & $8740(2)$ & $32(1)$ & 1 \\
\hline$C(5)$ & 14381(2) & 4191(1) & $9333(2)$ & $28(1)$ & 1 \\
\hline$C(6)$ & $13280(2)$ & 4581(1) & 9109(1) & $26(1)$ & 1 \\
\hline$C(7)$ & 11338(2) & 5868(1) & 7377(1) & $22(1)$ & 1 \\
\hline $\mathrm{C}(8)$ & $10389(2)$ & $6115(1)$ & 6669(1) & $29(1)$ & 1 \\
\hline C $(9)$ & $10589(2)$ & $6705(1)$ & $6231(2)$ & $37(1)$ & 1 \\
\hline $\mathrm{C}(10)$ & $11706(2)$ & 7060(1) & $6487(2)$ & $37(1)$ & 1 \\
\hline $\mathrm{C}(11)$ & $12635(2)$ & 6838(1) & 7192(2) & $34(1)$ & 1 \\
\hline$C(12)$ & $12458(2)$ & $6242(1)$ & 7641(2) & $29(1)$ & 1 \\
\hline$C(13)$ & $8569(2)$ & 3684(1) & 8144(1) & $21(1)$ & 1 \\
\hline$C(14)$ & $7365(2)$ & 3413(1) & 7947(1) & $27(1)$ & 1 \\
\hline$C(15)$ & $7165(2)$ & 2691(1) & $8037(2)$ & $33(1)$ & 1 \\
\hline$C(16)$ & $8149(2)$ & $2226(1)$ & $8309(2)$ & $36(1)$ & 1 \\
\hline$C(17)$ & 9354(2) & $2485(1)$ & $8501(1)$ & $30(1)$ & 1 \\
\hline $\mathrm{C}(18)$ & 9564(2) & $3203(1)$ & $8424(1)$ & $24(1)$ & 1 \\
\hline $\mathrm{C}(19)$ & 7343(2) & 5008(1) & 7514(1) & 21(1) & 1 \\
\hline $\mathrm{C}(20)$ & 6889(2) & $5165(1)$ & 6672(2) & $33(1)$ & 1 \\
\hline $\mathrm{C}(21)$ & $5683(2)$ & $5441(1)$ & $6328(2)$ & $39(1)$ & 1 \\
\hline$C(22)$ & 4971(2) & 5564(1) & 6842(2) & $34(1)$ & 1 \\
\hline$C(23)$ & $5430(2)$ & 5418(1) & 7683(2) & $33(1)$ & 1 \\
\hline $\mathrm{C}(24)$ & 6614(2) & $5135(1)$ & 8024(2) & $28(1)$ & 1 \\
\hline$C(25)$ & $9770(2)$ & 4142(1) & $6756(1)$ & $32(1)$ & 1 \\
\hline$C(26 \mathrm{~A})$ & 9747(3) & $4423(2)$ & $5930(2)$ & $29(1)$ & $0.606(4)$ \\
\hline $\mathrm{C}(27 \mathrm{~A})$ & 7688(4) & 4691(2) & $4830(2)$ & $46(1)$ & $0.606(4)$ \\
\hline $\mathrm{C}(26 \mathrm{~B})$ & 8771(5) & 4127(3) & $6040(3)$ & $28(1)$ & $0.394(4)$ \\
\hline$C(27 B)$ & $8508(6)$ & 5082(3) & 4772(4) & $38(2)$ & $0.394(4)$ \\
\hline $\mathrm{C}(31)$ & $3736(2)$ & $2840(1)$ & $5658(2)$ & 41(1) & 1 \\
\hline $\mathrm{Cl}(11)$ & 4592(1) & 3648(1) & $5838(1)$ & $53(1)$ & 1 \\
\hline $\mathrm{Cl}(12)$ & 2282(4) & 2929(2) & $5855(3)$ & $43(1)$ & $0.54(2)$ \\
\hline $\mathrm{Cl}(13)$ & $2215(5)$ & $3022(5)$ & $5595(12)$ & $90(2)$ & $0.46(2)$ \\
\hline C(32) & $3105(2)$ & 2924(2) & $1044(2)$ & $52(1)$ & 1 \\
\hline $\mathrm{Cl}(21)$ & 4595(1) & 2819(1) & 976(1) & 49(1) & 1 \\
\hline $\mathrm{Cl}(22)$ & 1989(2) & 2464(3) & 290(4) & $55(1)$ & $0.551(12)$ \\
\hline $\mathrm{Cl}(23)$ & 2129(3) & 2175(4) & $548(2)$ & $64(1)$ & $0.449(12)$ \\
\hline
\end{tabular}


Table 3. Selected bond lengths $[\AA ̊ \AA]$ and angles $\left[^{\circ}\right]$ for 5 (CCDC 258068).

\begin{tabular}{|c|c|c|c|}
\hline $\mathrm{Cr}(1)-\mathrm{Cl}(1)$ & $2.2701(5)$ & $\mathrm{Cl}(2)-\mathrm{Cr}(1)-\mathrm{Cl}(3) \# 1$ & $99.259(19)$ \\
\hline $\mathrm{Cr}(1)-\mathrm{Cl}(2)$ & $2.2900(5)$ & $\mathrm{Cl}(3)-\mathrm{Cr}(1)-\mathrm{Cl}(3) \# 1$ & $85.488(17)$ \\
\hline $\mathrm{Cr}(1)-\mathrm{Cl}(3)$ & $2.3679(5)$ & $\mathrm{Cl}(1)-\mathrm{Cr}(1)-\mathrm{P}(1)$ & $86.376(19)$ \\
\hline $\mathrm{Cr}(1)-\mathrm{Cl}(3) \# 1$ & $2.3939(5)$ & $\mathrm{Cl}(2)-\mathrm{Cr}(1)-\mathrm{P}(1)$ & $93.90(2)$ \\
\hline $\mathrm{Cr}(1)-\mathrm{P}(1)$ & $2.4251(6)$ & $\mathrm{Cl}(3)-\mathrm{Cr}(1)-\mathrm{P}(1)$ & $96.036(18)$ \\
\hline $\mathrm{Cr}(1)-\mathrm{P}(2)$ & $2.4862(6)$ & $\mathrm{Cl}(3) \# 1-\mathrm{Cr}(1)-\mathrm{P}(1)$ & $166.69(2)$ \\
\hline \multirow[t]{2}{*}{$\mathrm{Cl}(3)-\mathrm{Cr}(1) \# 1$} & $2.3939(5)$ & $\mathrm{Cl}(1)-\mathrm{Cr}(1)-\mathrm{P}(2)$ & $86.157(19)$ \\
\hline & & $\mathrm{Cl}(2)-\mathrm{Cr}(1)-\mathrm{P}(2)$ & $160.56(2)$ \\
\hline $\mathrm{Cl}(1)-\mathrm{Cr}(1)-\mathrm{Cl}(2)$ & $95.553(19)$ & $\mathrm{Cl}(3)-\mathrm{Cr}(1)-\mathrm{P}(2)$ & $87.510(18)$ \\
\hline $\mathrm{Cl}(1)-\mathrm{Cr}(1)-\mathrm{Cl}(3)$ & $171.71(2)$ & $\mathrm{Cl}(3) \# 1-\mathrm{Cr}(1)-\mathrm{P}(2)$ & $100.090(18)$ \\
\hline $\mathrm{Cl}(2)-\mathrm{Cr}(1)-\mathrm{Cl}(3)$ & $92.196(19)$ & $\mathrm{P}(1)-\mathrm{Cr}(1)-\mathrm{P}(2)$ & $66.837(18)$ \\
\hline $\mathrm{Cl}(1)-\mathrm{Cr}(1)-\mathrm{Cl}(3) \# 1$ & $90.364(18)$ & $\mathrm{Cr}(1)-\mathrm{Cl}(3)-\mathrm{Cr}(1) \# 1$ & $94.511(17)$ \\
\hline
\end{tabular}

Symmetry transformations used to generate equivalent atoms: $\# 1-x+2,-y+1,-z+2$

Table 4. Bond lengths $[\AA ̊ \cap]$ and angles $\left[^{\circ}\right]$ for 5 (CCDC 258068).

\begin{tabular}{llll}
\hline $\mathrm{Cr}(1)-\mathrm{Cl}(1)$ & $2.2701(5)$ & $\mathrm{C}(10)-\mathrm{C}(11)$ & $1.368(3)$ \\
$\mathrm{Cr}(1)-\mathrm{Cl}(2)$ & $2.2900(5)$ & $\mathrm{C}(10)-\mathrm{H}(10 \mathrm{~A})$ & $0.93(2)$ \\
$\mathrm{Cr}(1)-\mathrm{Cl}(3)$ & $2.3679(5)$ & $\mathrm{C}(11)-\mathrm{C}(12)$ & $1.387(3)$ \\
$\mathrm{Cr}(1)-\mathrm{Cl}(3) \# 1$ & $2.3939(5)$ & $\mathrm{C}(11)-\mathrm{H}(11 \mathrm{~A})$ & $0.88(2)$ \\
$\mathrm{Cr}(1)-\mathrm{P}(1)$ & $2.4251(6)$ & $\mathrm{C}(12)-\mathrm{H}(12 \mathrm{~A})$ & $0.92(2)$ \\
$\mathrm{Cr}(1)-\mathrm{P}(2)$ & $2.4862(6)$ & $\mathrm{C}(13)-\mathrm{C}(14)$ & $1.391(3)$ \\
$\mathrm{Cl}(3)-\mathrm{Cr}(1) \# 1$ & $2.3939(5)$ & $\mathrm{C}(13)-\mathrm{C}(18)$ & $1.397(3)$ \\
$\mathrm{P}(1)-\mathrm{N}(1)$ & $1.7010(16)$ & $\mathrm{C}(14)-\mathrm{C}(15)$ & $1.377(3)$ \\
$\mathrm{P}(1)-\mathrm{C}(7)$ & $1.8058(19)$ & $\mathrm{C}(14)-\mathrm{H}(14 \mathrm{~A})$ & $0.89(2)$ \\
$\mathrm{P}(1)-\mathrm{C}(1)$ & $1.8231(18)$ & $\mathrm{C}(15)-\mathrm{C}(16)$ & $1.367(3)$ \\
$\mathrm{P}(2)-\mathrm{N}(1)$ & $1.7089(15)$ & $\mathrm{C}(15)-\mathrm{H}(15 \mathrm{~A})$ & $0.91(2)$ \\
$\mathrm{P}(2)-\mathrm{C}(19)$ & $1.8141(18)$ & $\mathrm{C}(16)-\mathrm{C}(17)$ & $1.385(3)$ \\
$\mathrm{P}(2)-\mathrm{C}(13)$ & $1.8147(19)$ & $\mathrm{C}(16)-\mathrm{H}(16 \mathrm{~A})$ & $0.97(2)$ \\
$\mathrm{O}(1)-\mathrm{C}(27 \mathrm{~A})$ & $1.350(4)$ & $\mathrm{C}(17)-\mathrm{C}(18)$ & $1.369(3)$ \\
$\mathrm{O}(1)-\mathrm{C}(27 \mathrm{~B})$ & $1.361(6)$ & $\mathrm{C}(17)-\mathrm{H}(17 \mathrm{~A})$ & $0.87(2)$ \\
$\mathrm{O}(1)-\mathrm{C}(26 \mathrm{~A})$ & $1.415(3)$ & $\mathrm{C}(18)-\mathrm{H}(18 \mathrm{~A})$ & $0.855(18)$ \\
$\mathrm{O}(1)-\mathrm{C}(26 \mathrm{~B})$ & $1.491(5)$ & $\mathrm{C}(19)-\mathrm{C}(20)$ & $1.367(3)$ \\
$\mathrm{N}(1)-\mathrm{C}(25)$ & $1.488(2)$ & $\mathrm{C}(19)-\mathrm{C}(24)$ & $1.385(3)$ \\
$\mathrm{C}(1)-\mathrm{C}(6)$ & $1.390(3)$ & $\mathrm{C}(20)-\mathrm{C}(21)$ & $1.399(3)$ \\
$\mathrm{C}(1)-\mathrm{C}(2)$ & $1.391(3)$ & $\mathrm{C}(20)-\mathrm{H}(20 \mathrm{~A})$ & $0.88(2)$ \\
$\mathrm{C}(2)-\mathrm{C}(3)$ & $1.385(3)$ & $\mathrm{C}(21)-\mathrm{C}(22)$ & $1.373(3)$ \\
$\mathrm{C}(2)-\mathrm{H}(2 \mathrm{~A})$ & $0.89(2)$ & $\mathrm{C}(21)-\mathrm{H}(21 \mathrm{~A})$ & $0.92(2)$ \\
$\mathrm{C}(3)-\mathrm{C}(4)$ & $1.365(3)$ & $\mathrm{C}(22)-\mathrm{C}(23)$ & $1.361(3)$ \\
$\mathrm{C}(3)-\mathrm{H}(3 \mathrm{~A})$ & $0.84(2)$ & $\mathrm{C}(22)-\mathrm{H}(22 \mathrm{~A})$ & $0.89(2)$ \\
$\mathrm{C}(4)-\mathrm{C}(5)$ & $1.361(3)$ & $\mathrm{C}(23)-\mathrm{C}(24)$ & $1.383(3)$ \\
$\mathrm{C}(4)-\mathrm{H}(4 \mathrm{~A})$ & $0.86(2)$ & $\mathrm{C}(23)-\mathrm{H}(23 \mathrm{~A})$ & $0.86(2)$ \\
$\mathrm{C}(5)-\mathrm{C}(6)$ & $1.387(3)$ & $\mathrm{C}(24)-\mathrm{H}(24 \mathrm{~A})$ & $0.92(2)$ \\
$\mathrm{C}(5)-\mathrm{H}(5 \mathrm{~A})$ & $0.841(19)$ & $\mathrm{C}(25)-\mathrm{C}(26 \mathrm{~B})$ & $1.359(5)$ \\
$\mathrm{C}(6)-\mathrm{H}(6 \mathrm{~A})$ & $0.906(19)$ & $\mathrm{C}(25)-\mathrm{C}(26 \mathrm{~A})$ & $1.468(4)$ \\
$\mathrm{C}(7)-\mathrm{C}(12)$ & $1.389(3)$ & $\mathrm{C}(25)-\mathrm{H}(25 \mathrm{~A})$ & 0.9900 \\
$\mathrm{C}(7)-\mathrm{C}(8)$ & $1.397(3)$ & $\mathrm{C}(25)-\mathrm{H}(25 \mathrm{~B})$ & 0.9900 \\
$\mathrm{C}(8)-\mathrm{C}(9)$ & $1.376(3)$ & $\mathrm{C}(25)-\mathrm{H}(25 \mathrm{C})$ & 0.9900 \\
$\mathrm{C}(8)-\mathrm{H}(8 \mathrm{~A})$ & $0.91(2)$ & $\mathrm{C}(25)-\mathrm{H}(25 \mathrm{D})$ & 0.9901 \\
$\mathrm{C}(9)-\mathrm{C}(10)$ & $1.370(3)$ & $\mathrm{C}(26 \mathrm{~A})-\mathrm{H}(25 \mathrm{C})$ & 1.2145 \\
$\mathrm{C}(9)-\mathrm{H}(9 \mathrm{~A})$ & $0.86(2)$ & $\mathrm{C}(26 \mathrm{~A})-\mathrm{H}(26 \mathrm{~A})$ & 0.9900 \\
& & &
\end{tabular}


$-$

$\mathrm{C}(26 \mathrm{~A})-\mathrm{H}(26 \mathrm{~B})$

C(27A)-H(27A)

C(27A)-H(27B)

C(27A)-H(27C)

C(26B)-H(26C)

C(26B)-H(26D)

C(27B)-H(27D)

C(27B)-H(27E)

C(27B)-H(27F)

$\mathrm{C}(31)-\mathrm{Cl}(13)$

C(31)-Cl(11)

$\mathrm{C}(31)-\mathrm{Cl}(12)$

C(31)-H(31A)

C(31)-H(31B)

C(31)-H(31C)

C(31)-H(31D)

$\mathrm{C}(32)-\mathrm{Cl}(22)$

$\mathrm{C}(32)-\mathrm{Cl}(21)$

$\mathrm{C}(32)-\mathrm{Cl}(23)$

C(32)-H(32A)

C(32)-H(32B)

C(32)-H(32C)

C(32)-H(32D)

$\mathrm{Cl}(1)-\mathrm{Cr}(1)-\mathrm{Cl}(2)$

$\mathrm{Cl}(1)-\mathrm{Cr}(1)-\mathrm{Cl}(3)$

$\mathrm{Cl}(2)-\mathrm{Cr}(1)-\mathrm{Cl}(3)$

$\mathrm{Cl}(1)-\mathrm{Cr}(1)-\mathrm{Cl}(3) \# 1$

$\mathrm{Cl}(2)-\mathrm{Cr}(1)-\mathrm{Cl}(3) \# 1$

$\mathrm{Cl}(3)-\mathrm{Cr}(1)-\mathrm{Cl}(3) \# 1$

$\mathrm{Cl}(1)-\mathrm{Cr}(1)-\mathrm{P}(1)$

$\mathrm{Cl}(2)-\mathrm{Cr}(1)-\mathrm{P}(1)$

$\mathrm{Cl}(3)-\mathrm{Cr}(1)-\mathrm{P}(1)$

$\mathrm{Cl}(3) \# 1-\mathrm{Cr}(1)-\mathrm{P}(1)$

$\mathrm{Cl}(1)-\mathrm{Cr}(1)-\mathrm{P}(2)$

$\mathrm{Cl}(2)-\mathrm{Cr}(1)-\mathrm{P}(2)$

$\mathrm{Cl}(3)-\mathrm{Cr}(1)-\mathrm{P}(2)$

Cl(3)\#1-Cr(1)-P(2)

$\mathrm{P}(1)-\mathrm{Cr}(1)-\mathrm{P}(2)$

$\mathrm{Cr}(1)-\mathrm{Cl}(3)-\mathrm{Cr}(1) \# 1$

$\mathrm{N}(1)-\mathrm{P}(1)-\mathrm{C}(7)$

$\mathrm{N}(1)-\mathrm{P}(1)-\mathrm{C}(1)$

C(7)-P(1)-C(1)

$\mathrm{N}(1)-\mathrm{P}(1)-\mathrm{Cr}(1)$

C(7)-P(1)-Cr(1)

C(1)-P(1)-Cr(1)

$\mathrm{N}(1)-\mathrm{P}(2)-\mathrm{C}(19)$

$\mathrm{N}(1)-\mathrm{P}(2)-\mathrm{C}(13)$

$\mathrm{C}(19)-\mathrm{P}(2)-\mathrm{C}(13)$

$\mathrm{N}(1)-\mathrm{P}(2)-\mathrm{Cr}(1)$

$\mathrm{C}(19)-\mathrm{P}(2)-\mathrm{Cr}(1)$

C(13)-P(2)-Cr(1)

$\mathrm{C}(27 \mathrm{~A})-\mathrm{O}(1)-\mathrm{C}(27 \mathrm{~B})$

$\mathrm{C}(27 \mathrm{~A})-\mathrm{O}(1)-\mathrm{C}(26 \mathrm{~A})$

$\mathrm{C}(27 \mathrm{~B})-\mathrm{O}(1)-\mathrm{C}(26 \mathrm{~A})$

$\mathrm{C}(27 \mathrm{~A})-\mathrm{O}(1)-\mathrm{C}(26 \mathrm{~B})$

C(27B)-O(1)-C(26B)

\subsection{0 \\ 0.9800 \\ 0.9800 \\ 0.9800 \\ 0.9900 \\ 0.9900 \\ 0.9800 \\ 0.9800 \\ 0.9800 \\ 1.726(7) \\ $1.760(2)$ \\ $1.789(5)$ \\ 0.9900 \\ 0.9900 \\ 0.9901 \\ 0.9899 \\ 1.701(3) \\ 1.741(2) \\ 1.804(5) \\ 0.9900 \\ 0.9900 \\ 0.9899 \\ 0.9899}

95.553(19)

171.71(2)

92.196(19)

90.364(18)

99.259(19)

85.488(17)

86.376(19)

93.90(2)

96.036(18)

166.69(2)

86.157(19)

$160.56(2)$

$87.510(18)$

100.090(18)

66.837(18)

94.511(17)

108.42(9)

111.23(8)

105.09(8)

95.11(5)

117.67(6)

118.70(6)

112.39(8)

104.47(8)

103.70(9)

92.76(5)

$114.48(6)$

127.84(6)

52.9(3)

130.9(3)

114.9(3)

105.3(3)

143.2(3)
$\mathrm{C}(26 \mathrm{~A})-\mathrm{O}(1)-\mathrm{C}(26 \mathrm{~B})$

$\mathrm{C}(25)-\mathrm{N}(1)-\mathrm{P}(1)$

$\mathrm{C}(25)-\mathrm{N}(1)-\mathrm{P}(2)$

$\mathrm{P}(1)-\mathrm{N}(1)-\mathrm{P}(2)$

C(6)-C(1)-C(2)

C(6)-C(1)-P(1)

$\mathrm{C}(2)-\mathrm{C}(1)-\mathrm{P}(1)$

$\mathrm{C}(3)-\mathrm{C}(2)-\mathrm{C}(1)$

$\mathrm{C}(3)-\mathrm{C}(2)-\mathrm{H}(2 \mathrm{~A})$

$\mathrm{C}(1)-\mathrm{C}(2)-\mathrm{H}(2 \mathrm{~A})$

$\mathrm{C}(4)-\mathrm{C}(3)-\mathrm{C}(2)$

$\mathrm{C}(4)-\mathrm{C}(3)-\mathrm{H}(3 \mathrm{~A})$

$\mathrm{C}(2)-\mathrm{C}(3)-\mathrm{H}(3 \mathrm{~A})$

$\mathrm{C}(5)-\mathrm{C}(4)-\mathrm{C}(3)$

$\mathrm{C}(5)-\mathrm{C}(4)-\mathrm{H}(4 \mathrm{~A})$

$\mathrm{C}(3)-\mathrm{C}(4)-\mathrm{H}(4 \mathrm{~A})$

$\mathrm{C}(4)-\mathrm{C}(5)-\mathrm{C}(6)$

$\mathrm{C}(4)-\mathrm{C}(5)-\mathrm{H}(5 \mathrm{~A})$

$\mathrm{C}(6)-\mathrm{C}(5)-\mathrm{H}(5 \mathrm{~A})$

$\mathrm{C}(5)-\mathrm{C}(6)-\mathrm{C}(1)$

$\mathrm{C}(5)-\mathrm{C}(6)-\mathrm{H}(6 \mathrm{~A})$

$\mathrm{C}(1)-\mathrm{C}(6)-\mathrm{H}(6 \mathrm{~A})$

$\mathrm{C}(12)-\mathrm{C}(7)-\mathrm{C}(8)$

C(12)-C(7)-P(1)

$\mathrm{C}(8)-\mathrm{C}(7)-\mathrm{P}(1)$

$\mathrm{C}(9)-\mathrm{C}(8)-\mathrm{C}(7)$

$\mathrm{C}(9)-\mathrm{C}(8)-\mathrm{H}(8 \mathrm{~A})$

$\mathrm{C}(7)-\mathrm{C}(8)-\mathrm{H}(8 \mathrm{~A})$

$\mathrm{C}(10)-\mathrm{C}(9)-\mathrm{C}(8)$

$\mathrm{C}(10)-\mathrm{C}(9)-\mathrm{H}(9 \mathrm{~A})$

$\mathrm{C}(8)-\mathrm{C}(9)-\mathrm{H}(9 \mathrm{~A})$

C(11)-C(10)-C(9)

C(11)-C(10)-H(10A)

$\mathrm{C}(9)-\mathrm{C}(10)-\mathrm{H}(10 \mathrm{~A})$

C(10)-C(11)-C(12)

C(10)-C(11)-H(11A)

C(12)-C(11)-H(11A)

$\mathrm{C}(11)-\mathrm{C}(12)-\mathrm{C}(7)$

$\mathrm{C}(11)-\mathrm{C}(12)-\mathrm{H}(12 \mathrm{~A})$

C(7)-C(12)-H(12A)

C(14)-C(13)-C(18)

$\mathrm{C}(14)-\mathrm{C}(13)-\mathrm{P}(2)$

$\mathrm{C}(18)-\mathrm{C}(13)-\mathrm{P}(2)$

C(15)-C(14)-C(13)

C(15)-C(14)-H(14A)

C(13)-C(14)-H(14A)

C(16)-C(15)-C(14)

$\mathrm{C}(16)-\mathrm{C}(15)-\mathrm{H}(15 \mathrm{~A})$

$\mathrm{C}(14)-\mathrm{C}(15)-\mathrm{H}(15 \mathrm{~A})$

$\mathrm{C}(15)-\mathrm{C}(16)-\mathrm{C}(17)$

C(15)-C(16)-H(16A)

C(17)-C(16)-H(16A)

C(18)-C(17)-C(16)

C(18)-C(17)-H(17A)

$\mathrm{C}(16)-\mathrm{C}(17)-\mathrm{H}(17 \mathrm{~A})$

C(17)-C(18)-C(13)

C(17)-C(18)-H(18A)
$53.0(2)$

$126.65(13)$

124.37(13)

105.01(8)

118.74(18)

$117.86(15)$

123.37(16)

$119.7(2)$

$120.5(14)$

$119.8(14)$

120.7(2)

$123.7(17)$

115.6(17)

$120.3(2)$

117.8(16)

121.8(16)

120.2(2)

124.4(13)

$115.3(13)$

120.3(2)

120.2(12)

$119.5(12)$

118.79(19)

$118.95(15)$

121.93(16)

$120.0(2)$

121.1(13)

118.6(13)

120.7(2)

120.3(14)

$119.0(15)$

120.2(2)

$120.3(15)$

$119.5(14)$

120.1(2)

$122.6(14)$

117.2(14)

120.2(2)

121.3(13)

118.3(13)

118.30(18)

121.98(15)

119.72(14)

$120.7(2)$

120.9(13)

$118.4(13)$

120.4(2)

121.3(13)

118.2(13)

$119.8(2)$

121.7(12)

$118.5(12)$

$120.4(2)$

118.1(14)

$121.5(14)$

$120.5(2)$

119.8(12) 
$-$

$\mathrm{C}(13)-\mathrm{C}(18)-\mathrm{H}(18 \mathrm{~A})$

C(20)-C(19)-C(24)

$\mathrm{C}(20)-\mathrm{C}(19)-\mathrm{P}(2)$

$\mathrm{C}(24)-\mathrm{C}(19)-\mathrm{P}(2)$

C(19)-C(20)-C(21)

$\mathrm{C}(19)-\mathrm{C}(20)-\mathrm{H}(20 \mathrm{~A})$

$\mathrm{C}(21)-\mathrm{C}(20)-\mathrm{H}(20 \mathrm{~A})$

$\mathrm{C}(22)-\mathrm{C}(21)-\mathrm{C}(20)$

$\mathrm{C}(22)-\mathrm{C}(21)-\mathrm{H}(21 \mathrm{~A})$

$\mathrm{C}(20)-\mathrm{C}(21)-\mathrm{H}(21 \mathrm{~A})$

$\mathrm{C}(23)-\mathrm{C}(22)-\mathrm{C}(21)$

$\mathrm{C}(23)-\mathrm{C}(22)-\mathrm{H}(22 \mathrm{~A})$

$\mathrm{C}(21)-\mathrm{C}(22)-\mathrm{H}(22 \mathrm{~A})$

$\mathrm{C}(22)-\mathrm{C}(23)-\mathrm{C}(24)$

$\mathrm{C}(22)-\mathrm{C}(23)-\mathrm{H}(23 \mathrm{~A})$

$\mathrm{C}(24)-\mathrm{C}(23)-\mathrm{H}(23 \mathrm{~A})$

$\mathrm{C}(23)-\mathrm{C}(24)-\mathrm{C}(19)$

$\mathrm{C}(23)-\mathrm{C}(24)-\mathrm{H}(24 \mathrm{~A})$

$\mathrm{C}(19)-\mathrm{C}(24)-\mathrm{H}(24 \mathrm{~A})$

$\mathrm{C}(26 \mathrm{~B})-\mathrm{C}(25)-\mathrm{C}(26 \mathrm{~A})$

$\mathrm{C}(26 \mathrm{~B})-\mathrm{C}(25)-\mathrm{N}(1)$

$\mathrm{C}(26 \mathrm{~A})-\mathrm{C}(25)-\mathrm{N}(1)$

C(26B)-C(25)-H(25A)

C(26A)-C(25)-H(25A)

$\mathrm{N}(1)-\mathrm{C}(25)-\mathrm{H}(25 \mathrm{~A})$

$\mathrm{C}(26 \mathrm{~B})-\mathrm{C}(25)-\mathrm{H}(25 \mathrm{~B})$

$\mathrm{C}(26 \mathrm{~A})-\mathrm{C}(25)-\mathrm{H}(25 \mathrm{~B})$

$\mathrm{N}(1)-\mathrm{C}(25)-\mathrm{H}(25 \mathrm{~B})$

$\mathrm{H}(25 \mathrm{~A})-\mathrm{C}(25)-\mathrm{H}(25 \mathrm{~B})$

C(26B)-C(25)-H(25C)

$\mathrm{C}(26 \mathrm{~A})-\mathrm{C}(25)-\mathrm{H}(25 \mathrm{C})$

$\mathrm{N}(1)-\mathrm{C}(25)-\mathrm{H}(25 \mathrm{C})$

$\mathrm{H}(25 \mathrm{~A})-\mathrm{C}(25)-\mathrm{H}(25 \mathrm{C})$

$\mathrm{H}(25 \mathrm{~B})-\mathrm{C}(25)-\mathrm{H}(25 \mathrm{C})$

$\mathrm{C}(26 \mathrm{~B})-\mathrm{C}(25)-\mathrm{H}(25 \mathrm{D})$

C(26A)-C(25)-H(25D)

$\mathrm{N}(1)-\mathrm{C}(25)-\mathrm{H}(25 \mathrm{D})$

$\mathrm{H}(25 \mathrm{~A})-\mathrm{C}(25)-\mathrm{H}(25 \mathrm{D})$

$\mathrm{H}(25 \mathrm{~B})-\mathrm{C}(25)-\mathrm{H}(25 \mathrm{D})$

$\mathrm{H}(25 \mathrm{C})-\mathrm{C}(25)-\mathrm{H}(25 \mathrm{D})$

$\mathrm{O}(1)-\mathrm{C}(26 \mathrm{~A})-\mathrm{C}(25)$

$\mathrm{O}(1)-\mathrm{C}(26 \mathrm{~A})-\mathrm{H}(25 \mathrm{C})$

$\mathrm{O}(1)-\mathrm{C}(26 \mathrm{~A})-\mathrm{H}(26 \mathrm{~A})$

C(25)-C(26A)-H(26A)

$\mathrm{H}(25 \mathrm{C})-\mathrm{C}(26 \mathrm{~A})-\mathrm{H}(26 \mathrm{~A})$

$\mathrm{O}(1)-\mathrm{C}(26 \mathrm{~A})-\mathrm{H}(26 \mathrm{~B})$

$\mathrm{C}(25)-\mathrm{C}(26 \mathrm{~A})-\mathrm{H}(26 \mathrm{~B})$

$\mathrm{H}(25 \mathrm{C})-\mathrm{C}(26 \mathrm{~A})-\mathrm{H}(26 \mathrm{~B})$

$\mathrm{H}(26 \mathrm{~A})-\mathrm{C}(26 \mathrm{~A})-\mathrm{H}(26 \mathrm{~B})$

$\mathrm{O}(1)-\mathrm{C}(27 \mathrm{~A})-\mathrm{H}(27 \mathrm{~A})$

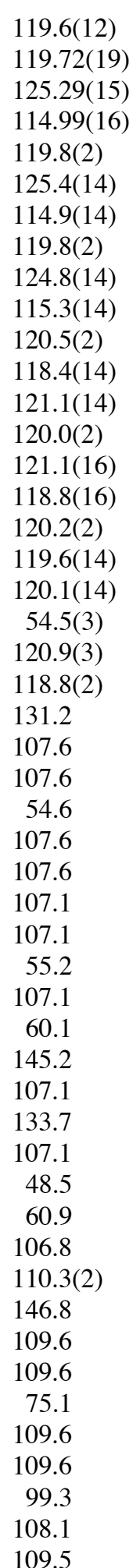

$\mathrm{O}(1)-\mathrm{C}(27 \mathrm{~A})-\mathrm{H}(27 \mathrm{~B}) \quad 109.5$

$\mathrm{O}(1)-\mathrm{C}(27 \mathrm{~A})-\mathrm{H}(27 \mathrm{C}) \quad 109.5$

$\mathrm{C}(25)-\mathrm{C}(26 \mathrm{~B})-\mathrm{O}(1) \quad 112.2(4)$

$\mathrm{C}(25)-\mathrm{C}(26 \mathrm{~B})-\mathrm{H}(26 \mathrm{C}) \quad 109.2$

$\mathrm{O}(1)-\mathrm{C}(26 \mathrm{~B})-\mathrm{H}(26 \mathrm{C}) \quad 109.2$

$\mathrm{C}(25)-\mathrm{C}(26 \mathrm{~B})-\mathrm{H}(26 \mathrm{D}) \quad 109.2$

$\mathrm{O}(1)-\mathrm{C}(26 \mathrm{~B})-\mathrm{H}(26 \mathrm{D}) \quad 109.2$

$\mathrm{H}(26 \mathrm{C})-\mathrm{C}(26 \mathrm{~B})-\mathrm{H}(26 \mathrm{D}) \quad 107.9$

$\mathrm{O}(1)-\mathrm{C}(27 \mathrm{~B})-\mathrm{H}(27 \mathrm{D}) \quad 109.5$

$\mathrm{O}(1)-\mathrm{C}(27 \mathrm{~B})-\mathrm{H}(27 \mathrm{E}) \quad 109.5$

$\mathrm{H}(27 \mathrm{D})-\mathrm{C}(27 \mathrm{~B})-\mathrm{H}(27 \mathrm{E}) \quad 109.5$

$\mathrm{O}(1)-\mathrm{C}(27 \mathrm{~B})-\mathrm{H}(27 \mathrm{~F}) \quad 109.5$

$\mathrm{H}(27 \mathrm{D})-\mathrm{C}(27 \mathrm{~B})-\mathrm{H}(27 \mathrm{~F}) \quad 109.5$

$\mathrm{H}(27 \mathrm{E})-\mathrm{C}(27 \mathrm{~B})-\mathrm{H}(27 \mathrm{~F}) \quad 109.5$

$\mathrm{Cl}(13)-\mathrm{C}(31)-\mathrm{Cl}(11) \quad 109.2(3)$

$\mathrm{Cl}(11)-\mathrm{C}(31)-\mathrm{Cl}(12) \quad 112.35(16)$

$\mathrm{Cl}(13)-\mathrm{C}(31)-\mathrm{H}(31 \mathrm{~A}) \quad 122.6$

$\mathrm{Cl}(11)-\mathrm{C}(31)-\mathrm{H}(31 \mathrm{~A}) \quad 109.1$

$\mathrm{Cl}(12)-\mathrm{C}(31)-\mathrm{H}(31 \mathrm{~A}) \quad 109.1$

$\mathrm{Cl}(13)-\mathrm{C}(31)-\mathrm{H}(31 \mathrm{~B}) \quad 97.9$

$\mathrm{Cl}(11)-\mathrm{C}(31)-\mathrm{H}(31 \mathrm{~B}) \quad 109.1$

$\mathrm{Cl}(12)-\mathrm{C}(31)-\mathrm{H}(31 \mathrm{~B}) \quad 109.1$

$\mathrm{H}(31 \mathrm{~A})-\mathrm{C}(31)-\mathrm{H}(31 \mathrm{~B}) \quad 107.9$

$\mathrm{Cl}(13)-\mathrm{C}(31)-\mathrm{H}(31 \mathrm{C}) \quad 112.3$

$\mathrm{Cl}(11)-\mathrm{C}(31)-\mathrm{H}(31 \mathrm{C}) \quad 110.5$

$\mathrm{Cl}(12)-\mathrm{C}(31)-\mathrm{H}(31 \mathrm{C}) \quad 121.6$

$\mathrm{H}(31 \mathrm{~A})-\mathrm{C}(31)-\mathrm{H}(31 \mathrm{C}) \quad 91.8$

$\mathrm{Cl}(13)-\mathrm{C}(31)-\mathrm{H}(31 \mathrm{D}) \quad 105.6$

$\mathrm{Cl}(11)-\mathrm{C}(31)-\mathrm{H}(31 \mathrm{D}) \quad 110.4$

$\mathrm{Cl}(12)-\mathrm{C}(31)-\mathrm{H}(31 \mathrm{D}) \quad 91.4$

$\mathrm{H}(31 \mathrm{~B})-\mathrm{C}(31)-\mathrm{H}(31 \mathrm{D}) \quad 123.2$

$\mathrm{H}(31 \mathrm{C})-\mathrm{C}(31)-\mathrm{H}(31 \mathrm{D}) \quad 108.7$

$\mathrm{Cl}(22)-\mathrm{C}(32)-\mathrm{Cl}(21) \quad 113.71(16)$

$\mathrm{Cl}(21)-\mathrm{C}(32)-\mathrm{Cl}(23) \quad 110.42(18)$

$\mathrm{Cl}(22)-\mathrm{C}(32)-\mathrm{H}(32 \mathrm{~A}) \quad 108.8$

$\mathrm{Cl}(21)-\mathrm{C}(32)-\mathrm{H}(32 \mathrm{~A}) \quad 108.8$

$\mathrm{Cl}(23)-\mathrm{C}(32)-\mathrm{H}(32 \mathrm{~A}) \quad 90.6$

$\mathrm{Cl}(22)-\mathrm{C}(32)-\mathrm{H}(32 \mathrm{~B}) \quad 108.8$

$\mathrm{Cl}(21)-\mathrm{C}(32)-\mathrm{H}(32 \mathrm{~B}) \quad 108.8$

$\mathrm{Cl}(23)-\mathrm{C}(32)-\mathrm{H}(32 \mathrm{~B}) \quad 127.8$

$\mathrm{H}(32 \mathrm{~A})-\mathrm{C}(32)-\mathrm{H}(32 \mathrm{~B}) \quad 107.7$

$\mathrm{Cl}(22)-\mathrm{C}(32)-\mathrm{H}(32 \mathrm{C}) \quad 88.9$

$\mathrm{Cl}(21)-\mathrm{C}(32)-\mathrm{H}(32 \mathrm{C}) \quad 109.6$

$\mathrm{Cl}(23)-\mathrm{C}(32)-\mathrm{H}(32 \mathrm{C}) \quad 109.9$

$\mathrm{H}(32 \mathrm{~A})-\mathrm{C}(32)-\mathrm{H}(32 \mathrm{C}) \quad 125.7$

$\mathrm{Cl}(22)-\mathrm{C}(32)-\mathrm{H}(32 \mathrm{D}) \quad 124.3$

$\mathrm{Cl}(21)-\mathrm{C}(32)-\mathrm{H}(32 \mathrm{D}) \quad 109.5$

$\mathrm{Cl}(23)-\mathrm{C}(32)-\mathrm{H}(32 \mathrm{D}) \quad 109.4$

$\mathrm{H}(32 \mathrm{~B})-\mathrm{C}(32)-\mathrm{H}(32 \mathrm{D}) \quad 87.9$

H(32C)-C(32)-H(32D) 108.1

Symmetry transformations used to generate equivalent atoms: \#1 -x+2,-y+1,-z+2 
Table 5. Anisotropic displacement parameters $\left(\AA^{2} x 1^{4}\right)$ for 5 (CCDC 258068). The anisotropic displacement factor exponent takes the form: $-2 \pi^{2}\left[h^{2} a^{* 2} U^{11}+\ldots+2 h k a^{*} b^{*}\right.$ $\left.\mathbf{U}^{12}\right]$

\begin{tabular}{|c|c|c|c|c|c|c|}
\hline & $\mathrm{U}^{11}$ & $\mathrm{U}^{22}$ & $\mathrm{U}^{33}$ & $U^{23}$ & $\mathrm{U}^{13}$ & $\mathrm{U}^{12}$ \\
\hline $\operatorname{Cr}(1)$ & $146(1)$ & $168(1)$ & $161(2)$ & $-8(1)$ & $54(1)$ & $10(1)$ \\
\hline $\mathrm{Cl}(1)$ & $200(2)$ & $197(2)$ & $221(2)$ & $23(2)$ & $67(2)$ & $44(2)$ \\
\hline $\mathrm{Cl}(2)$ & $179(2)$ & $222(2)$ & $269(3)$ & $-15(2)$ & $35(2)$ & $-19(2)$ \\
\hline $\mathrm{Cl}(3)$ & $179(2)$ & $194(2)$ & $176(2)$ & $5(2)$ & $73(2)$ & $32(2)$ \\
\hline $\mathrm{P}(1)$ & $183(2)$ & $193(2)$ & 201(3) & $20(2)$ & $91(2)$ & $34(2)$ \\
\hline $\mathrm{P}(2)$ & $171(2)$ & 201(2) & $177(3)$ & $-24(2)$ & $55(2)$ & $4(2)$ \\
\hline $\mathrm{O}(1)$ & $413(9)$ & $553(10)$ & $206(9)$ & $-80(7)$ & $-12(7)$ & $202(8)$ \\
\hline $\mathrm{N}(1)$ & $196(8)$ & $237(8)$ & $180(8)$ & $-47(7)$ & $69(6)$ & $7(6)$ \\
\hline $\mathrm{C}(1)$ & 186(9) & $169(9)$ & $288(11)$ & $59(8)$ & 131(8) & $31(7)$ \\
\hline $\mathrm{C}(2)$ & $300(12)$ & $319(12)$ & $324(14)$ & $21(10)$ & $163(10)$ & $62(9)$ \\
\hline $\mathrm{C}(3)$ & $315(12)$ & $296(12)$ & $492(16)$ & $-33(11)$ & $241(12)$ & $66(9)$ \\
\hline $\mathrm{C}(4)$ & 197(11) & $229(11)$ & $556(16)$ & $75(10)$ & $170(11)$ & $48(9)$ \\
\hline $\mathrm{C}(5)$ & $192(10)$ & $311(11)$ & $347(14)$ & $91(10)$ & $84(10)$ & $0(9)$ \\
\hline$C(6)$ & $225(10)$ & $266(10)$ & $328(13)$ & $25(9)$ & $141(9)$ & $8(8)$ \\
\hline$C(7)$ & $250(10)$ & 219(9) & $233(11)$ & $13(8)$ & $147(8)$ & $64(8)$ \\
\hline $\mathrm{C}(8)$ & $358(13)$ & $282(11)$ & $235(12)$ & 19(9) & $97(10)$ & $45(10)$ \\
\hline C(9) & $537(16)$ & $308(12)$ & 251(13) & $74(10)$ & $126(12)$ & $139(11)$ \\
\hline$C(10)$ & $590(16)$ & $247(11)$ & $409(15)$ & $115(10)$ & $336(13)$ & $109(11)$ \\
\hline $\mathrm{C}(11)$ & $364(13)$ & $264(11)$ & $495(16)$ & $81(10)$ & $263(12)$ & $29(10)$ \\
\hline$C(12)$ & $252(11)$ & $279(11)$ & $370(13)$ & $86(10)$ & $159(10)$ & 67(9) \\
\hline $\mathrm{C}(13)$ & $250(10)$ & 200(9) & $173(10)$ & $-59(8)$ & $54(8)$ & $-25(8)$ \\
\hline$C(14)$ & $247(11)$ & $278(11)$ & $270(12)$ & $-48(9)$ & $73(9)$ & $-7(9)$ \\
\hline$C(15)$ & $317(12)$ & $299(12)$ & $387(14)$ & $-113(10)$ & $112(10)$ & $-124(10)$ \\
\hline$C(16)$ & $480(15)$ & $240(11)$ & $329(13)$ & $-57(10)$ & $93(11)$ & $-88(10)$ \\
\hline $\mathrm{C}(17)$ & $323(12)$ & $238(11)$ & 294(13) & $-31(9)$ & $36(10)$ & 21(9) \\
\hline $\mathrm{C}(18)$ & 241(11) & $235(10)$ & $212(11)$ & $-38(8)$ & $33(8)$ & $-21(8)$ \\
\hline$C(19)$ & $159(9)$ & 181(9) & $272(11)$ & $-40(8)$ & $51(8)$ & 4(7) \\
\hline $\mathrm{C}(20)$ & $268(12)$ & $442(13)$ & $304(13)$ & $27(10)$ & $112(10)$ & $113(10)$ \\
\hline $\mathrm{C}(21)$ & $327(13)$ & $473(14)$ & $342(15)$ & $113(12)$ & $54(11)$ & $105(11)$ \\
\hline $\mathrm{C}(22)$ & $178(11)$ & $277(11)$ & $544(16)$ & $-10(10)$ & $107(10)$ & $36(9)$ \\
\hline $\mathrm{C}(23)$ & $216(11)$ & $377(12)$ & $433(15)$ & $-89(11)$ & $149(10)$ & $-7(9)$ \\
\hline$C(24)$ & $215(10)$ & $363(12)$ & $280(13)$ & $-68(10)$ & $90(9)$ & $-26(9)$ \\
\hline$C(25)$ & $435(13)$ & $322(11)$ & $240(12)$ & $-49(9)$ & $145(10)$ & $91(10)$ \\
\hline$C(26 \mathrm{~A})$ & $330(20)$ & $340(20)$ & $185(18)$ & $-30(14)$ & $65(15)$ & 131(16) \\
\hline$C(27 \mathrm{~A})$ & $420(20)$ & $670(30)$ & $280(20)$ & $-130(20)$ & $117(19)$ & $-120(20)$ \\
\hline$C(26 B)$ & $300(30)$ & $270(30)$ & $280(30)$ & $-70(20)$ & $100(20)$ & $-30(20)$ \\
\hline$C(27 B)$ & $400(40)$ & $480(40)$ & $280(30)$ & $60(30)$ & $120(30)$ & $90(30)$ \\
\hline $\mathrm{C}(31)$ & $435(14)$ & $302(12)$ & $447(15)$ & $-52(11)$ & $94(12)$ & $53(10)$ \\
\hline $\mathrm{Cl}(11)$ & $696(4)$ & $395(3)$ & $501(4)$ & $-26(3)$ & $207(3)$ & $-103(3)$ \\
\hline $\mathrm{Cl}(12)$ & $305(12)$ & $390(15)$ & $557(17)$ & $-125(9)$ & $86(10)$ & $-39(10)$ \\
\hline $\mathrm{Cl}(13)$ & $435(14)$ & $990(30)$ & $1050(60)$ & $-380(30)$ & $-90(20)$ & $275(17)$ \\
\hline $\mathrm{C}(32)$ & $420(15)$ & 601(17) & 495(17) & $-85(14)$ & $90(12)$ & 141(13) \\
\hline $\mathrm{Cl}(21)$ & $384(3)$ & $423(3)$ & $689(5)$ & $85(3)$ & $228(3)$ & $46(3)$ \\
\hline $\mathrm{Cl}(22)$ & $310(8)$ & $610(20)$ & $690(20)$ & $-305(15)$ & $121(9)$ & 2(9) \\
\hline $\mathrm{Cl}(23)$ & $412(10)$ & $666(19)$ & $692(14)$ & 210(17) & $-12(9)$ & $66(11)$ \\
\hline
\end{tabular}

